\title{
Open Heavy Flavor Theory
}

\author{
Vincenzo Greco ${ }^{a, b, *}$ \\ ${ }^{a}$ Department of Physics and Astronomy, University of Catania, \\ Via S. Sofia 64, Catania, Italy \\ ${ }^{b}$ Istituto Nazionale di Fisica Nucleare INFN-LNS, \\ Via S. Sofia 62, Catania, Italy
}

E-mail: greco@lns.infn.it

We briefly discuss the different aspects that enters the open heavy flavor physics focusing the recent developments and the upcoming issues that can be tackled. It has become clear that the interaction of heavy quarks in the Hot QCD matter is largely non-perturbative and the phenomenological estimates within still large error bars are in agreement with the lattice QCD calculation. However there are still significant source of uncertainty that are expected to be reduced thanks to the new and up coming data at low momenta, new observables and the access to bottom measurement. Furthermore a new aspect that is attracting more and more interest is the non-expected large heavy baryon production. More recently, it is growing also the investigation of the role that heavy quarks can have as a probe of the initial stage of the collisions: impact of glasma dynamics, vorticity and electromagnetic fields.

HardProbes 2020

1-6 June 2020

Austin, Texas

${ }^{*}$ Speaker 


\section{Introduction}

A main probe of the properties of the Quark-Gluon Plasma (QGP) is constituted by the heavy quarks, namely the charm and bottom. They are considered "heavy" because their mass scale is quite larger than $\Lambda_{Q C D}$, but it is also quite larger than the temperature reached in ultra-relativistic heavy-ion collisions (uRHICs), $m_{b, c}>>T$. The hard scale of the mass, $m_{b, c}>>\Lambda_{Q C D}$, implies that one can also compute the $p_{T}$ spectra of the particle in pp collision and within the uncertainty due to shadowing effects give the baseline of the production in pA and AA. There have been extensive measurements of HQ production in elementary collisions that demonstrate their production is indeed calculable in perturbative QCD at least at moderate and high $p_{T}$ within next-to-next-to-leading order (NNLO) perturbative QCD schemes like the Fixed-Flavour-Number Scheme (FFNS), where the heavy quark is not an active parton in the proton, the General Mass Variable Fixed Number Scheme (GV-VFNS) where the number of active flavours is increased when the regularization scale cross the mass of the HQ or the Fixed Order Next Leading Logarithm (FONLL) based on the matching of the massive NLO cross section in the FFNS with the massless NLO calculation in the zero mass VNFS; for these studies one can refer to Ref. [1] and the references therein. However, only very recently, as presented in this Conference, the pT spectra of $D^{0}$ mesons has been measured by ALICE and CMS down to a fraction of $\mathrm{GeV}$ and at a quite high precision that now at low momenta is smaller than the estimated theoretical uncertainties, see [2]. So we have now an excellent knowledge of what should be the intial distribution of HQ in AA collisions modulus the effect of shadowing.

The scale hierarchy $m_{b, c}>>T$ implies that there is no thermal production (even if for charm at top LHC energy or future FCC collider this may be not enough accurate) and hence HQs are produced at $\tau_{0} \sim 1 / 2 m_{b, c}<0.1 \mathrm{fm} / \mathrm{c}$ and then undergo the entire QGP dynamics. A further hierachy is $m_{b, c}>g T$ which implies that the momentum exchange with the bulk Hot QCD matter is small and the motion is of Brownian nature; this hiearchy scale may be challenged in a strongly interacting medium $(g \sim 2-3)$ for charm quarks [3], but still remain true that a drag and diffusion coefficients determine the charm quark dynamics at least at low momenta region. This feature is quite relevant because it also allow to scrutiny if the transport coefficient that can be inferred from the phenomenological studies are in agreement with the calculation from lattice QCD [4]. Indeed a further advantage of HQs is the possibility to derive also a Non-Relativistic QCD approach, see [5].

\section{Current stage and envisaged developments for the determination of $D_{s}(T)$}

A first goal of the studies of open heavy flavor observables has been the determination of the drag and diffusion transport coeffcient that drive the change in the average momentum of the HQ and the width of the fluctuations around such an average momentum. From a phenomenological point of view this has been conducted by developing models able to link the value of the drag and diffusion to two main observables, namely the nuclear modification factor $R_{A A}\left(p_{T}\right)$ and the elliptic flow $v_{2}$. More recently there has been also first comparisons to the measurement of higher order anisotropic flow, mainly the triangular one $v_{3}\left(p_{T}\right)$. Such studies have led to the conclusion that certainly the interaction of HQ is largely non-perturbative and the value of the space diffusion 
coefficient $D_{s} \simeq 2-5 / 2 \pi T$ at $T \sim T_{c}$, close to the expectations from AdS/CFT, but with a moderate temperature dependence, as shown in Fig. 1.

In the last few years there have been several joint activities among theoriticians and experimentalists to clarify the sources of uncertainties toward a determination of the HQ transport coefficients [13-15]. A significant source of uncertainty comes from the extrapolation $p \rightarrow 0$ needed to compare to the IQCD calculation according to the proper definition of $D_{s}$. The phenomenological approaches till now have been based on the comparison to experimental data on $R_{A A}\left(p_{T}\right)$ and $v_{2}\left(p_{T}\right)$ in the range $p_{T} \sim 2-6 \mathrm{GeV}$. It happens that the different models have similar value of the drag and diffusion at intermediate $p_{T}$ region, but present larger differences in the $p \rightarrow 0$ limit. A significant source of uncertainty is the impact of the hadronization process. Already some

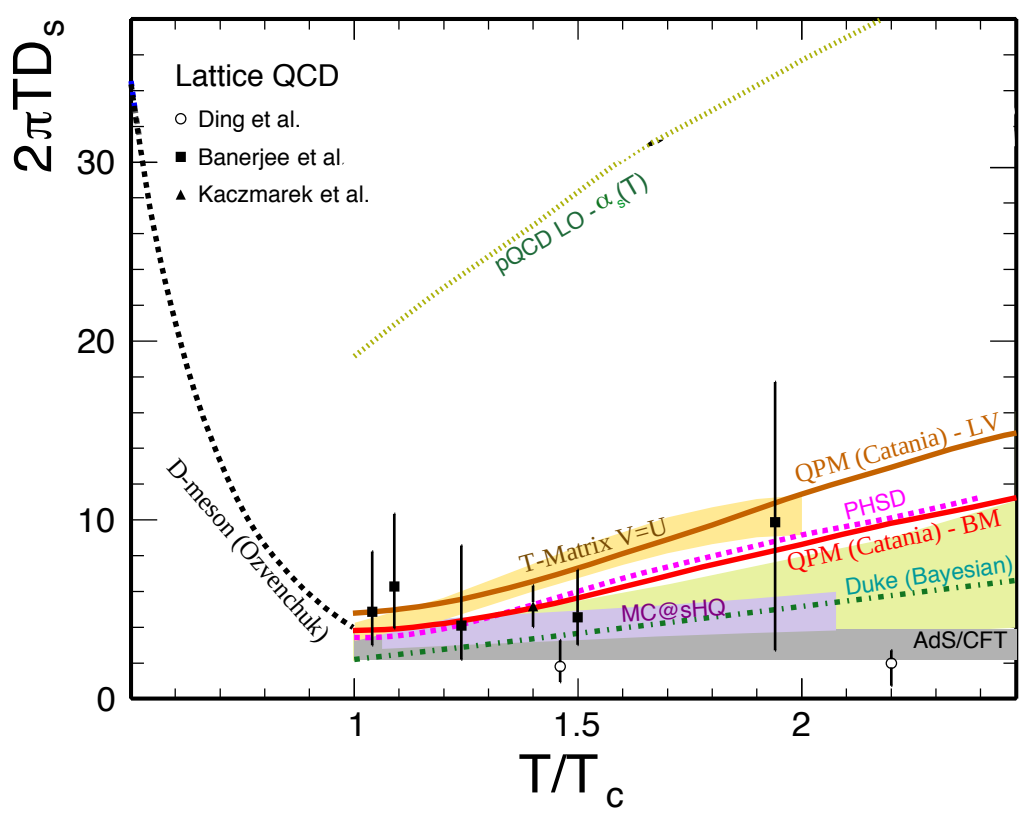

Figure 1: (Color online) Charm-quark diffusion coefficients from quenched 1QCD calculations [6] (squares) [7] (triangles) and [8] (open circles) compared to model calculations based on different elastic interactions in the QGP: QPM calculations with a Boltzmann (BM) dynamics and with a Langevin (LV) one [9], PHSD transport calculation [22], T -matrix approach [10], MC@sHQ perturbative approach [11], and AdS/CFT estimate. The yellow band shows a Bayesian analysis fit result based on the Duke dynamic model [12]. Also shown on the figure are the Ds coefficients for D-mesons in hadronic matter.

years ago it has been recognized that a coalescence process leads to an enhancement of both the $R_{A A}\left(p_{T}\right)$ and $v_{2}\left(p_{T}\right)$, a feature needed by all the models. However a direct comparison shows that different approaches lead quantitavely to significant differences [13]. The access to the data not only for $\mathrm{D}$ mesons, but also to $\Lambda_{c}$ should allow in principle to constraint better the impact of hadronization, see also Sect. 4. A minor source of uncertainty still not negligible has been, till now, the employed initial charm distribution at low $p_{T}$ which is associated to the increasing uncertainty in next-to-leading order expansion. However with the new data on D meson this aspect can be overcome starting from a fit to the experimental distribution that is measured down to quite low $p_{T}$. A further moderate source of uncertainty in the case of charm comes from the assumed transport dynamics: a Boltzmann dynamics leads to estimate a $D_{S}(T)$ about a $20-30 \%$ smaller w.r.t. to 
the assumption of pure Brownian motion, see Fig. 1. A source of uncertainty that should nearly disappear for the bottom dynamics. It has been emphasized that also the assumed hydrodynamical bulk evolution can represent a source of uncertainty, an issue that however can in principle asymptotically be overcome exploiting the advances in the determination of the bulk matter evolution with the most recent models with $3 \mathrm{D}+1$ viscous hydrodynamics. The current upgrade that allows to access the low momentum region significantly reducing the error bars will certainly allow a more solid quantitative determination of the transport coefficients for HQ in Hot QCD matter. To this will add the upcoming availability of the first data for $\mathrm{B}$ mesons at intermediate $p_{T}$ that can further constraint theoretical models. The open heavy flavor study is also entering a stage in which will be possible to access more exclusive observables.

The phenomenological approach is extending the study to event-by-event investigations studying not only higher harmonics like $v_{3,4}[16,17]$, but also the correlation between the $v_{n}$ of heavy quarks and that of the charged bulk hadrons which should be quite sensitive to the temperature dependence of $D_{s}(T)$ [18]. These are going to be accessible also experimentally.

\section{Most recent developments}

There are several developments in the last 1-2 years in both the theorethical and phenomenological approaches. From the point of view of the initial production in NLO pQCD, a higher dimensional expansion has been developed, that allows not only the $p_{T}$ distribution, but also angolar correlations between the HQs pairs [19]. This is a key development for the study of triggered $D-h$ and $D-\bar{D}$ angular correlation in both pA and AA collisions.

A key approach to study the microscopic dynamics of the charm quarks has been the T-matrix that starting from the data of lattice QCD for the free energy of $Q \bar{Q}$ infers the elastic scattering cross section for $q(\bar{q}) Q$. A missing aspect has been the self-consistent inclusion of the radiative energy loss. In Ref. [31] the first development in this direction has been presented showing that due to the large finite mass the radiation is quite suppressed, but still close to $T_{c}$ the remants of confinement partially counter balance such a suppression. The impact on the observables has to be seen.

An approach that has been developed in the context of pQCD to study mainly the high- $p_{T}$ region is DREENA developed initially in a $1+1 \mathrm{D}$ geometry providing solid predicition for $R_{A A}$, very recently it has been extended to 3+1D. This allows for a realistic study of both $R_{A A}$ and $v_{2}$ for $\pi, B, D$ mesons; first results shows some tension for a simultaneous prediction of both observables [20] that is however milder than other models. Furthermore it has been suggested that the ratio $v_{2} /\left(1-R_{A A}\right)$ high $p_{T}$ provide a solid constraint of the intial eccentricity.

A further issue that has to be investigated is the impact of initial non-equilibrium due to nonthermal anisotropies in the pressure and energy/density ratio but also to the non-equilibrium in the dynamical properties of the interaction that may lead to shift in the pole masses. A first study in [21] shows mild effect in AA collisions that may become more relevant in pA collisions.

The interaction of HQ is largely non-perturbative which can be expected to induce a sizeable width of the quasi-particle exicitation of the bulk QGP. However the off-shell dynamics is discarded essentially in all approaches that are able to realistically predicted HQs observales with the exception of PHSD [22]. The effect of off-shell dynamics on the HQ transport coefficient has been studied some years ago [23], now in Ref. [32] a first direct comparison of the impact of off-shell dynamics 
in the energy loss spectrum for a charm quark has been done finding that for width $\Gamma \lesssim M_{q, g}$ the impact on the $R_{A A}\left(p_{T}\right)$ is quite small.

A general key issue in the understanding of the matter created in uRHICs is the system size dependence. It has been suggested that a system size scan in AA such that one observes how the elliptic flow of charm quark changes decreasing the system size by a factor of 2 while simultaneously increasing the eccentricity by a similar factor can shed light on the mechanism in action in $\mathrm{pPb}$. Theoretically in the presence of a QGP one should observe a scaling of the elliptic flow $v_{2}\left(p_{T}\right)$ [24].

\section{Hadronization}

In the first stage of the theoretical models of the D meson production in AA collision, it has been implicitly assumed that $\Lambda_{c}$ production is negligible. In fact from $e^{+}+e^{-}, e^{+}+p$ collisions and even in $p p$ collisions at LHC (at forward rapidity) it was estimated that the fraction $f\left(c \rightarrow \Lambda_{c}\right)$ of charm fragmenting into $\Lambda_{c}$ is 0.06 . Similarly also prediction of Monte Carlo codes like PYTHIA were supplying similar prediction for $\Lambda_{c} / D$ also at mid-rapidity. However there was a prediction that according to hadronization by coalescence the $\Lambda_{c} / D$ can reach values of $O(1)$ at mid-rapidity [25]. More recently it has been noted that a color reconnection mechanism can lead to a large enhancement of the baryon production.
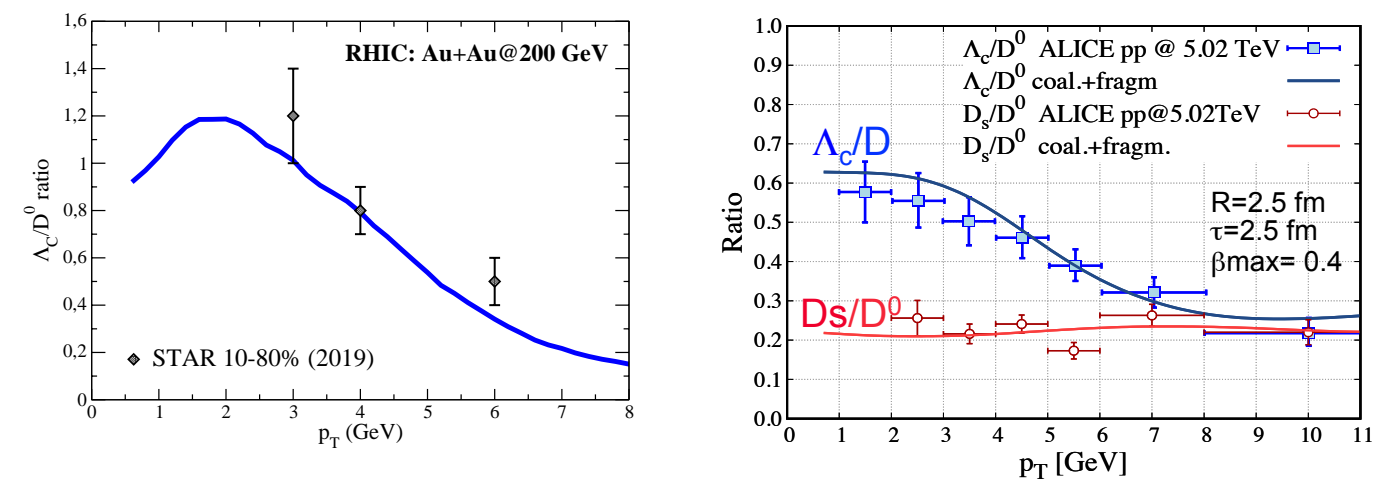

Figure 2: (Color online) - Left : $\Lambda_{c} / D$ ratio vs $p_{T}$ in AA collisions at RHIC energy [27] compared to a coalescence plus fragmention model [28]. Right: $\Lambda_{c} / D$ and $D_{s} / D$ vs $p_{T}$ in pp collisions compared to a model coalescence plus fragmention model [30], see text for more details.

In Fig. 2 (left) we can see the recent measurement in AA collisions at both RHIC and LHC energy along with the predictions from a coalescence plus fragmentation model [28], see also [26]. Such a large production of $\Lambda_{c}$ would indeed affect also the model of the evaluation of $D$ meson $R_{A A}\left(p_{T}\right)$. This is an aspect that in general has not been taken into account into theoretical approaches and its impact should be evaluated in the next years. More generally this finding is opening up a new topic within the open heavy flavor physics that is the hadronization mechanism for heavy baryon in high energy nuclear collisions. In fact if it is true that the large ratio is observed in AA collision and may be related to a coalescence mechanism it is also true that similar observations 
have been done by ALICE also in $p A$ and even $p p$ collisions [29]. In Fig. 2 (right) it is shown the $\Lambda_{c} / D$ and $D_{s} / D$ in $p p$ collisions assuming that a mini QGP fireball is formed according to viscous hydrodynamics simulations [30].

In this conference it has been shown that several other heavy charm baryon resonances can be measured and hence there are abundant new experimental data that can allow to get a new insight into the hadronization mechanism. The large baryon production appears as a large effect coming from the presence of a dense medium both in a color reconnection and coalescence approach. Therefore the charm baryon production will be an interesting physical question to investigate also at the EIC facility.

\section{Heavy quark in the initial stage: glasma, vorticity and electromagnetic fields}

In recent years it has become evident that the matter created in uRHICs has a very large vorticity and in the intial stage undergoes huge electromagnetic fields. Furthermore, in the initial stage, it is expected that glasma matter is created with chromomagnetic fields that quickly decay into the QGP. A frontier that is opening up is to employ HQs as a probe of such intial stage. This has led to first studies of the impact of the initial non-equilibrium glasma matter on the HQs dynamics, an aspect till now neglected. The very high energy density of the glasma seems to induce a large diffusion that dominates over the drag effect. As shown in Fig. 3 (left), this can lead to a first stage of the dynamics $(\tau \lesssim 0.3 \mathrm{fm} / \mathrm{c})$ where the HQ $p_{T}$ spectrum is not quenched, but oppositely to what happens for a particle-like interaction in the QGP. This could account for the $R_{p P b}$ at intermediate $p_{T}$ and modify the initial time evolution of the momentum spectrum in AA collisions [35]. A first preliminary study would indicate that this mechanism would imply a somewhat larger interaction in the QGP phase to account for the measured $R_{A A}\left(p_{T}\right)$ and implies also an increase of the $v_{2}\left(p_{T}\right)$ of about a $15 \%$ [36]. A self consistent and more complete study is however to be pursued.
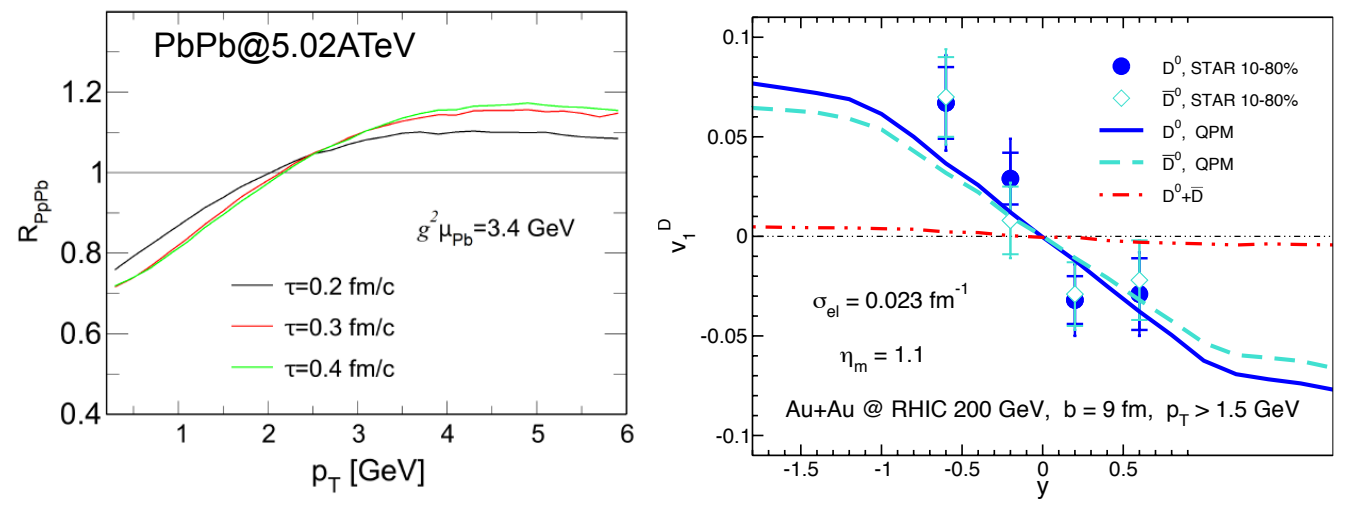

Figure 3: (Color online) - Left: Evolution of the $R_{A A}\left(p_{T}\right)$ in a dynamical approach based on glasma dynamics under condition similar to $P b+P b$ collisions [35]. Right: directed flow of $\mathrm{D}$ mesons assuming a pQCD interaction for charm quarks (red dashed-dot line) and the non-perturbative Quasi-Particle-Model (blu lines) that includes also the impact of the electromagnetic field compared to STAR experimental data [38]. 
Another new aspect has been the prediction of a large transverse flow $v_{1}=<p_{x} / p_{T}>$ for HQs. Contrary to a naive prediction that would assume that the $v_{1}$ should be smaller than the one of the light hadron, in Ref. [37]. it was predicted that, due to the tilted distribution of the bulk associated to the large vorticity and angular momentum of the matter created, the charm quark can acquire a $v_{1}$ that is at least one order of magnitude larger. First experimental data from STAR and also from ALICE has shown that this is indeed the case finding a value about 25 times larger for D meson w.r.t. to pion and kaons. In Ref. [40] it has been shown that within the same models employed for the determination of the $D_{s}(T)$ one can correctly predict the large $v_{1}(y)$ and assuming a tilted distribution of the bulk in agreement with the study on $\Lambda$ polarization one can quantitively predict the measured $v_{1}$. It is also seen that such a large $v_{1}$ is also another manifestation of the non-perturbation HQ interaction; in Fig. 3, we show the $v_{1}$ predicted with the Quasi Particle Model (QPM) (blue lines) that accounts for the non-perturbative interaction and the results assuming a pQCD HQ interaction (red line) along with the experimental data by STAR . Furthermore in Ref. [34] it has been pointed out that the HQs can be a probe also of the intial large electromagnetic field that induces a splitting of $v_{1}$ between $D^{0}$ and $\bar{D}^{0}$, an effect that seems to be observed by STAR [38] and ALICE [39], even if with still large error bars. Very recently, it has been proposed the measurment of the $v_{1}$ of the leptons from $Z^{0}$ decay that should show a more clear signal of the intial e-m. fields and clarify the origin of the observed splitting for D mesons [41].

\section{References}

[1] A. Andronic, et al. Eur. Phys. J. C 76 (2016) no.3, 107

[2] C. Mironov, in these Proceedings.

[3] S. K. Das, F. Scardina, S. Plumari and V. Greco, Phys. Rev. C 90 (2014), 044901

[4] X. Dong and V. Greco, Prog. Part. Nucl. Phys. 104 (2019), 97-141

[5] N. Brambilla, in these Proceedings.

[6] D. Banerjee, S. Datta, R. Gavai and P. Majumdar, Phys. Rev. D 85 (2012), 014510

[7] O. Kaczmarek, Nucl. Phys. A 931 (2014), 633-637

[8] H. T. Ding, F. Karsch and S. Mukherjee, Int. J. Mod. Phys. E 24 (2015) no.10, 1530007

[9] F. Scardina, S. K. Das, V. Minissale, S. Plumari and V. Greco, Phys. Rev. C 96 (2017) n. 4, 044905

[10] M. He, R. J. Fries and R. Rapp, Phys. Rev. C 86 (2012), 014903

[11] P. B. Gossiaux, J. Aichelin, B. Guiot, I. Karpenko, V. Ozvenchuk, T. Pierog, J. Steinheimer and K. Werner, PoS HardProbes2018 (2019), 169

[12] Y. Xu, J. E. Bernhard, S. A. Bass, M. Nahrgang and S. Cao, Phys. Rev. C 97 (2018) no.1, 014907 
[13] R. Rapp, P. B. Gossiaux, A. Andronic, R. Averbeck, S. Masciocchi, A. Beraudo, E. Bratkovskaya, P. Braun-Munzinger, et al. Nucl. Phys. A 979 (2018), 21-86

[14] S. Cao, et al. Phys. Rev. C 99 (2019) no.5, 054907.

[15] Y. Xu, S. A. Bass, et al. Phys. Rev. C 99 (2019) no.1, 014902

[16] M. Nahrgang, J. Aichelin, S. Bass, P. B. Gossiaux and K. Werner, Phys. Rev. C 91 (2015) no. 1,014904

[17] A. Beraudo, A. De Pace, M. Monteno, M. Nardi and F. Prino, JHEP 02 (2018), 043

[18] S. Plumari, G. Coci, V. Minissale, S. K. Das, Y. Sun and V. Greco, Phys. Lett. B 805 (2020), 135460

[19] R. Vogt, in these Proceedings.

[20] D. Zigic, in these Proceedings.

[21] T. Song, P. Moreau, Y. Xu, V. Ozvenchuk, E. Bratkovskaya, J. Aichelin, S. A. Bass, P. B. Gossiaux and M. Nahrgang, Phys. Rev. C 101 (2020) no.4, 044903

[22] T. Song, H. Berrehrah, D. Cabrera, J. M. Torres-Rincon, L. Tolos, W. Cassing and E. Bratkovskaya, Phys. Rev. C 92 (2015) no.1, 014910

[23] H. Berrehrah, E. Bratkovskaya, W. Cassing, P. B. Gossiaux, J. Aichelin and M. Bleicher, Phys. Rev. C 89 (2014) no.5, 054901

[24] J. Noronha-Hostler, in these Proceedings.

[25] Y. Oh, C. M. Ko, S. H. Lee and S. Yasui, Phys. Rev. C 79 (2009), 044905

[26] Shan Shan Cao, in these Proceedings.

[27] J. Adam et al. [STAR], Phys. Rev. Lett. 124 (2020) no.17, 172301

[28] S. Plumari, V. Minissale, S. K. Das, G. Coci and V. Greco, Eur. Phys. J. C 78 (2018) no.4, 348

[29] C. Hills, in these Proceedings.

[30] V. Minissale, S. Plumari and V. Greco, in preparation.

[31] S. Y. F. Liu and R. Rapp, JHEP 08 (2020), 168

[32] M. L. Sambataro, S. Plumari and V. Greco, [arXiv:2005.14470 [hep-ph]].

[33] S. K. Das, F. Scardina, S. Plumari and V. Greco, Phys. Lett. B 747 (2015), 260-264

[34] S. K. Das, S. Plumari, S. Chatterjee, J. Alam, F. Scardina and V. Greco, Phys. Lett. B 768 (2017), 260-264 
[35] J. H. Liu, S. Plumari, S. K. Das, V. Greco and M. Ruggieri, Phys. Rev. C 102 (2020) no.4, 044902

[36] Y. Sun, S. K. Das, S. Plumari, M. Ruggieri and V. Greco, Phys. Lett. B 798 (2019), 134933

[37] S. Chatterjee and P. Bożek, Phys. Rev. Lett. 120 (2018) no.19, 192301

[38] J. Adam et al. [STAR], Phys. Rev. Lett. 123 (2019) no.16, 162301

[39] S. Acharya et al. [ALICE], Phys. Rev. Lett. 125 (2020) no.2, 022301

[40] L. Oliva, S. Plumari and V. Greco, [arXiv:2009.11066 [hep-ph]].

[41] Y. Sun, S. Plumari and V. Greco, [arXiv:2004.09880 [nucl-th]]. 